\title{
Un enfoque davidsoniano de los delirios: el caso del delirio de Capgras
}

\author{
Emilia Vilatta \\ Consejo Nacional de Investigaciones Científicas y Técnicas (CONICET) - \\ Universidad Nacional de Córdoba
}

\begin{abstract}
Resumen: Recientemente, algunos críticos del enfoque davidsoniano de la atribución intencional han señalado que el mismo no puede ser aplicado para el caso de los delirios psiquiátricos, dado que las creencias delirantes no satisfacen los requisitos de racionalidad que este impone. En este trabajo: i) reconstruyo, a partir del análisis del caso del delirio de Capgras, la crítica a la idea de que solo podemos interpretar a un agente con creencias irracionales si mantiene aún un trasfondo de racionalidad; ii) objeto la misma y argumento que este delirio no representa un verdadero contraejemplo ya que un examen adecuado del mismo muestra que los sujetos con delirio de Capgras conservan un trasfondo de racionalidad. Señalaré así, que las condiciones mínimas para que la atribución de estados intencionales tenga lugar se encuentran garantizadas.
\end{abstract}

Palabras clave: delirios; síndrome de Capgras; atribución intencional; Donald Davidson; racionalidad

Abstract: "A Davidsonian Approach to Delusions: the Case of the Capgras
Delusion". Recently, some critics of Davidson's approach of intentional attribution
have pointed out that such a view cannot be applied to psychiatric delusions, since
delusional beliefs do not satisfy the rational requirements it imposes. In this paper:
i) I analyze, based on the Capgras syndrome case analysis, the objections to the
idea that an agent can only be interpreted as having irrational beliefs against a
background of rationality; ii) I object this criticism and I argue that this delusion
does not represent a true counterexample to Davidson's requirements, since an
appropriate evaluation shows that subjects with Capgras syndrome still preserve
a background of rationality. Thus, I will conclude that in such cases the minimum
conditions for attributing intentional states are guaranteed. Keywords: delusions; Capgras syndrome; intentional ascription; Donald Davidson; rationality 


\section{Introducción}

Los puntos de vista de Donald Davidson sobre la interpretación de la mente y la acción están siendo discutidos con mayor frecuencia en la reciente literatura de la filosofia de la psiquiatría. Su enfoque racionalista sobre la atribución de estados intencionales ha sido particular y ampliamente debatido y criticado en recientes trabajos filosóficos sobre el fenómeno de los delirios psiquiátricos ${ }^{1}$.

En el contexto del debate en torno a la atribución intencional, Davidson ha sostenido que no es posible individuar los contenidos mentales atribuidos a otro sujeto, a menos que se realice en el marco de una amplia red de estados mentales intencionales adecuadamente relacionados entre $\mathbf{s i}^{2}$. Esto se debe a que, para el filósofo, el contenido de un estado mental particular depende de los vínculos inferenciales con otros contenidos. Asimismo, considera que para interpretar la conducta de otro agente en términos intencionales debemos suponer que la mayoria de sus creencias no son contradictorias entre sí y que los contenidos de sus creencias más básicas están constituidos por determinados rasgos objetivos básicos del entorno que han de ser verdaderos y compartidos. En virtud de esto, una interpretación intencional exitosa inviste necesariamente al agente interpretado de una racionalidad básica.

El consenso entre quienes discuten las ideas de Davidson en el ámbito de la filosofia de la psiquiatría resulta claro: el enfoque davidsoniano, según el cual la racionalidad es constitutiva de la atribución de estados mentales

\footnotetext{
1 Estas críticas pueden hallarse, por ejemplo, en los siguientes trabajos: Bortolotti, L., "Intentionality Without Rationality", en: Proceedings of the Aristotelian Society, v. CV (2005), pp. 369-376; Bortolotti, L., "Delusions and the Background of Rationality", en: Mind and Language, v. XX (2005), pp. 189-208; Campbell, J., "What Does Rationality Have to Do with Psychological Causation?”, en: Broome, M. y L. Bortolotti (eds.), Propositional Attitudes as Mechanisms and as Control Variables, Oxford: Oxford University Press, 2009, pp. 137-149; Klee, R., "Why Some Delusions Are Necessarily Inexplicable Beliefs", en: Philosophy, Psychiatry \& Psychology, v. XI (2004), pp. 25-34 y Gerrans, P., "Cognitive Architecture and the Limits of Interpretationism", en: Philosophy, Psychiatry, \& Psychology, v. XI (2009), pp. 43-48.

2 Esta idea puede encontrarse en numerosos escritos de Davidson: $c f$. Davidson, D., Subjective, Intersubjective Objective, Oxford: Oxford Claredon Press, 2001, pp. 95-105; "Incoherence and Irrationality", en: Dialectica, v. XXXIX (1985), pp. 345-354; "A Coherence Theory of Truth and Knowledge”, en: Lepore, E. (ed.), Perspectives in the Philosophy of Donald Davidson, Oxford: Basil Blackwell, 1986, pp. 307-319; "How is Weakness of the Will Possible?", en: Feinberg, J. (ed.), Moral Concepts, Oxford: Oxford University Press, 1970 y Problems of Rationality, Nueva York: Clarendon Press, 2004.
} 
intencionales, se ve socavado por el fenómeno de los delirios psiquiátricos. La razón que sustenta tal afirmación es la siguiente: las creencias delirantes parecen no satisfacer los requisitos de racionalidad impuestos por Davidson. Por ejemplo, en cuanto al requisito de coherencia, un paciente cree que puede controlar el clima a través de órdenes mentales personales, a pesar de poseer también creencias sobre ciertos hechos básicos de la meteorología que entran en contradicción con el primer estado doxástico. Otro paciente cree que su oreja izquierda es un segundo vientre fértil, a pesar de aceptar ciertos hechos básicos sobre la fertilidad, la concepción y la gestación que volverian imposible tal hecho ${ }^{3}$. En cuanto al requisito de correspondencia, por ejemplo, se ha señalado que los pacientes delirantes han sido muchas veces descritos -tanto por psiquiatras como por filósofos- como sujetos que "viven en otro mundo" o que "viven en mundo de sueños" por tener pensamientos fantásticos, a veces bizarros e incompresibles, y alejados del mundo real"4.

Tales descripciones, a juicio de los criticos, sugieren una profunda ausencia de los requisitos de racionalidad impuestos por Davidson para que podamos considerar a quienes sufren de delirios como agentes intencionales. Sin embargo, arguyen, parece evidente que los sujetos delirantes tienen estados intencionales con contenidos mentales, aunque estos últimos puedan resultarnos bizarros y/o alejados de la realidad. Por lo tanto, concluyen que la concepción davidsoniana debe estar equivocada o, en otras palabras, que la atribución de estados mentales intencionales no requiere necesariamente la satisfacción del requisito de racionalidad especificado por Davidson.

En el presente trabajo me concentraré en la crítica que ha realizado Lisa Bortolotti en contra de la concepción davidsoniana de la atribución de estados intencionales 5 . A mi juicio, esta resulta especialmente controvertida dado que niega la constricción de racionalidad como necesaria para la atribución de estados intencionales ${ }^{6}$.

3 Cf. Klee, R., "Why Some Delusions Are Necessarily Inexplicable Beliefs", pp. 25-34.

4 Cf. Scarone, S. y otros, "The Dream as a Model for Psychosis: an Experimental Approach Using Bizarreness as a Cognitive Marker", en: Schizophrenia Bulletin, v. XXXIV (2008), pp. 515-522.

5 Esta crítica a Davidson ha sido delineada por Bortolotti en los siguientes escritos: "Inconsistency and Interpretation", en: Philosophical Explorations, v. VI (2003), pp.109-123; "Can We Interpret Irrational Behavior?.”, en: Behavior and Philosophy, v. XXXII (2004), pp. 359-375; "Intentionality without Rationality", en: Proceedings of the Aristotelian Society, v. CV (2005), pp. 369-376; "Delusions and the Background of Rationality", en: Mind and Language, v. XX (2005), pp. 189-208 y Delusions and Other Irrational Beliefs, Nueva York: Oxford University Press, 2009.

6 Esta es la conclusión a la que arriba Lisa Bortolotti como consecuencia de una estrategia más amplia dirigida a defender una versión doxástica de los delirios (es decir, una que asuma que los delirios deben ser vistos como creencias). Dicha estrategia, esbozada en su libro Delusions 
Con el objetivo general de desafiar la concepción racionalista davidsoniana, Bortolotti ataca el holismo de lo mental defendido por Davidson centrándose particularmente en un argumento que este filósofo ha propuesto en sus escritos sobre la irracionalidad ${ }^{7}$. $\mathrm{El}$ argumento, denominado por Bortolotti como el "argumento del trasfondo", le permite a Davidson conciliar la existencia de fenómenos irracionales con su teoría de lo mental.

La estrategia central de Bortolotti consiste en defender lo que ella denomina la "tesis de la continuidad", según la cual no habría diferencias categóricas entre las creencias delirantes y las creencias ordinarias, al menos en lo que a la irracionalidad se refiere. Para apoyar esta idea, la autora se propone mostrar que muchas creencias ordinarias son bastante irracionales y que, dado que las creencias delirantes también lo son, en su opinión solo habría una diferencia de grado entre ambas ${ }^{8}$. Estoy de acuerdo con la tesis de la continuidad, pero por razones diferentes a las que Bortolotti ofrece. Mi estrategia procederá de modo inverso: intentaré mostrar que los delirios no son tan irracionales después de todo, y que el trasfondo de racionalidad en el patrón de estados mentales

and Other Irrational Beliefs (2009) busca confrontar un tipo de argumento que los defensores del no-doxasticismo utilizan a menudo: partir del supuesto de que la racionalidad es una característica esencial de las creencias para concluir que las ideas delirantes son demasiado irracionales para ser calificadas como genuinos estados doxásticos. A menudo, los defensores de la visión no doxástica no ofrecen argumentos propios para afirmar que la racionalidad es constitutiva de las creencias. Antes bien, suelen apoyarse en teorias previas que han defendido esta afirmación, tales como la de Davidson (Cf. "Two Paradoxes of Irrationality", en: Wollheim, R. y J. Hopkins (eds.), Philosophical Essays on Freud, Cambridge: Cambridge University Press, 1982, pp. 289-305) o Dennett ( $C f$. The Intentional Stance, Cambridge: MIT Press, 1987). Es en este contexto que Bortolotti se ve tentada a negar los supuestos racionalistas de la teoria de Davidson, ya que intenta preservar una versión doxástica de los delirios. Al respecto, cabe aclarar que no discutiré aquî -pues esto merecería un análisis aparte- la naturaleza de los delirios (es decir si es más adecuada una versión doxástica o no-doxástica sobre los mismos) y, en consonancia con Bortolotti, asumiré -al menos provisoriamente- que los mismos son creencias (Cf. Bortolotti, L., Delusions and Other Irrational Beliefs, Nueva York: Oxford University Press, 2009, p.3).

7 Cf. Davidson, D., "How is Weakness of the Will Possible?", en: Feinberg, J. (ed.), Moral Concepts, Oxford: Oxford University Press, 1970, pp. 93-113; Davidson, D., "Two Paradoxes of Irrationality", en: Wollheim, R. y J. Hopkins (eds.), Philosophical Essays on Freud, Cambridge: Cambridge University Press, 1982, pp. 289-305; Davidson, D., "Incoherence and Irrationality", en: Dialectica, v. XXXIX (1985), pp. 345-354 y Problems of Rationality, Nueva York: Clarendon Press, 2004. Si 186 bien en todos estos textos Davidson se ocupa de fenómenos tales como la debilidad de la voluntad ("akrasia") y el pensamiento ilusorio ("wishful thinking"), es decir casos de irracionalidad ordinarios o de la vida cotidiana, en el presente trabajo extenderé este enfoque a casos de irracionalidad del ámbito de la psicopatología.

8 A partir de experimentos en psicología cognitiva, Bortolotti compara las supuestas faltas de racionalidad de los delirios (que los defensores de la visión no-doxástica han esgrimido como razones a su favor) y ofrece ejemplos de creencias ordinarias que tendrian las mismas características relevantes. Pretende señalar así que tanto las creencias delirantes como las ordinarias son irracionales y que solo guardan diferencias de grado entre sí ( $C f$. Bortolotti, L., Delusions and Other Irrational Beliefs). 
y comportamientos de estos sujetos puede considerarse conservado. Cabe aclarar que el alcance de esta afirmación se restringirá a los tipos de delirios que Bortolotti analiza en sus escritos: delirios monotemáticos ${ }^{9}$. Asimismo, en oposición a Bortolotti, reafirmaré el argumento trascendental davidsoniano según el cual para atribuir intencionalidad es preciso atribuir racionalidad.

A tal efecto, el artículo presenta la siguiente estructura: en la primera parte reconstruiré el argumento del trasfondo que esgrime Davidson para dar cuenta de las atribuciones de estados mentales irracionales; en la segunda, me concentraré en las objeciones que ha desarrollado Bortolotti a este argumento y señalaré algunos problemas que, a mi criterio, la misma presenta; en la tercera, presentaré un caso de síndrome de Capgras para evaluar qué tipo de información contaría como evidencia legitima en contra de la creencia delirante del sujeto, y argumentaré que si consideramos el carácter peculiar de la experiencia del sujeto veremos que aunque parezca irracional no incurre en una violación explícita de racionalidad; finalmente, y de modo independiente, argumentaré que la tesis de la compartimentación de lo mental de Davidson puede permitirnos lidiar con este tipo de delirios y presentaré algunas consideraciones sobre por qué el trasfondo de racionalidad puede considerarse conservado en estos casos. A partir de dichas consideraciones, concluiré que esta clase de delirios no representa un contraejemplo al argumento del trasfondo esbozado por Davidson. Esto me permitirá afirmar que las condiciones mínimas para que la atribución de estados intencionales a sujetos delirantes tenga lugar se encuentran garantizadas, al menos en este tipo de fenómenos psicopatológicos.

\section{Holismo davidsoniano}

Davidson considera que solo es posible atribuir un estado mental con un contenido específico en el marco de una densa red de otros estados mentales relacionados adecuadamente entre sí, ya que el contenido de un estado mental particular depende de los vínculos inferenciales con otros $\operatorname{contenidos}^{10}$. Por

9 Los delirios monotemáticos son aquellos que se hallan circunscriptos a un tema particular. En contraposición, los delirios politemáticos son aquellos que pueden referir a más de una temática.

${ }^{10}$ Estas consideraciones pueden hallarse en numerosos textos de Davidson: $c f$. "Truth and Meaning”, en: Synthese, v. XVII (1967), pp. 304-323; “Thought and Talk", en: Guttenplan, S. (ed.), Mind and Language, Oxford: Oxford University Press, 1975, pp. 154-170; "A Coherence Theory of Truth and Knowledge", en: Henrich, D. (ed.), Kant oder Hegel?, Stuttgart: Klett-Cotta, 1983, pp. 428-438; "A Coherence Theory of Truth and Knowledge", en: Lepore, E. (ed.), Perspectives in the Philosophy of Donald Davidson, Oxford: Basil Blackwell, 1986, pp. 307-319; Mente, mundo y acción: claves para una interpretación, Barcelona: Paidós, 1992; Inquiries into Truth and Inter- 
ejemplo, para creer que mi perro está sentado sobre el sillón, debo tener muchas creencias verdaderas sobre los perros y sobre los sillones, sobre mi perro en particular y sobre mi sillón en particular, sobre el sitio y el momento en el que se encuentran, etcétera. Es decir, que siempre precisamos de un gran número de creencias verdaderas para cada pensamiento particular, más allá de que no sea posible especificar una lista fija de creencias para cada pensamiento.

Esta tesis holista se extiende del ámbito del pensamiento al de las acciones intencionales, las cuales se caracterizan distintivamente porque: i) pueden ser explicadas apelando a las creencias y deseos causantes de las mismas y ii) los contenidos proposicionales de las creencias y deseos mencionados en (i) racionalizan dichas acciones. Asimismo, en conformidad con el principio de caridad, debemos suponer que la mayoría de las creencias del agente no son contradictorias entre sí y que los contenidos de sus creencias más básicas están constituidos por determinados rasgos objetivos básicos del entorno, que han de ser verdaderos y compartidos ${ }^{11}$. Sin embargo, ¿qué sucede en aquellos casos en los cuales identificamos pensamientos irracionales en un agente? ¿Es posible atribuir esos estados mentales irracionales y al mismo tiempo -según el principio de caridad-considerar que el agente es racional? Consciente de esta tensión, Davidson brinda algunas consideraciones respecto a por qué la tesis holista sobre el pensamiento sigue siendo válida aún en estos casos, y la atribución puede realizarse sin mayores inconvenientes.

\section{I.1. El argumento del trasfondo}

Este argumento es presentado por Davidson en sus escritos sobre la irracionalidad, cuando intenta conciliar su enfoque racionalista del pensamiento -en particular el holismo de lo mental- con la existencia de fenómenos irracionales ${ }^{12}$.

Al hablar de irracionalidad, Davidson se refiere a determinados pensamientos o comportamientos que no encajan plenamente con el resto de las actitudes intencionales del agente, las cuales deben exhibir un trasfondo de racionalidad. Ningún estado particular per se, por ejemplo una creencia determinada -no

pretation, Oxford: Clarendon Press, 2001 y Subjective, Intersubjective Objective, Oxford: Oxford Claredon Press, 2001, pp. 95-105.

11 Cf. Davidson, D., Essays on Actions and Events, Nueva York: Oxford University Press, 1980, p. 238

${ }^{12}$ Cf. Davidson, D., "Two Paradoxes of Irrationality", en: Wollheim, R. y J. Hopkins (eds.), Philosophical Essays on Freud, Cambridge: Cambridge University Press, 1982, pp. 289-305; "Incoherence and Irrationality", en: Dialectica, v. XXXIX (1985), pp. 345-354 y Problems of Rationality, Nueva York: Clarendon Press, 2004, pp. 190-196. 
importa lo extraña que pueda resultarle a los demás-, puede ser considerada en sí misma como irracional. Como bien señala Davidson, las creencias "nunca son irracionales en sí mismas, sino dentro de un patrón más amplio"13.

Al respecto, el argumento del trasfondo sostiene que solo podemos interpretar a un agente como teniendo creencias irracionales siempre que el mismo mantenga aún un trasfondo de racionalidad en el resto de sus estados mentales. Esto es así ya que solo podremos dar sentido a tales creencias (e incluso a la designación de las mismas como "irracionales") por referencia a los demás estados mentales y acciones del sujeto, los cuales -según el principio de caridad-deben poder ser vistos como siguiendo las normas de racionalidad. No solo por preservar la inteligibilidad sino porque además, como señala Davidson, "sin el elemento de racionalidad, nos negamos a aceptar la explicación como apropiada para un fenómeno mental" ${ }^{14}$. Así, de acuerdo a Davidson, la tesis holista previamente mencionada valdría también para las creencias irracionale ${ }^{15}$ ya que somos capaces de identificar que un agente tiene una creencia irracional particular cuando podemos detectar además una red más amplia de creencias, deseos y actitudes vinculados inferencialmente a tal creencia, que cuenta con un trasfondo de racionalidad.

\section{Objeciones en contra del argumento del trasfondo}

En diversos trabajos, Bortolotti ${ }^{16}$ ha desafiado la tesis davidsoniana según la cual solo se pueden atribuir legitimamente estados mentales intencionales a un sujeto si este es ampliamente racional, es decir, si posee una red de estados mentales interconectados entre sí y con nuestras acciones mediante vínculos de racionalidad (holismo de lo mental). Como mencionamos previamente, Bortolotti objeta el argumento del trasfondo y defiende la idea de que algunos casos de delirios revelan la inadecuación de aquellas teorías de la interpretación que descansan sobre una constricción necesaria de racionalidad sobre la atribución de creencias. En sus palabras: "en particular, desafio el punto de

13 Ibid., p.142. La traducción es mía.

14 Ibid., p. 190.

15 Ibid., p. 196.

${ }^{16}$ Cf. Bortolotti, L., "Inconsistency and Interpretation", en: Philosophical Explorations, v. VI (2003), pp.109-123; "Can We Interpret Irrational Behavior?.", en: Behavior and Philosophy, v. XXXII (2004), pp. 359-375; "Intentionality without Rationality", en: Proceedings of the Aristotelian Society, v. CV (2005), pp. 369-376; "Delusions and the Background of Rationality", en: Mind and Language, v. XX (2005), pp. 189-208 y Delusions and Other Irrational Beliefs, Nueva York: Oxford University Press, 2009. 
vista de que las creencias irracionales solo pueden ser atribuidas en contra un trasfondo general de racionalidad. Los sujetos afectados por delirios parecen ser genuinos creyentes y sus creencias pueden ser exitosamente explicadas en términos intencionales, pero estos sujetos no cumplen con los criterios que según Davidson deben cumplir para que el trasfondo de racionalidad esté en su lugar" ${ }^{17}$. Ahora bien, ¿cómo procede Bortolotti a fin de cuestionar el argumento del trasfondo? Su argumentación puede ser sintetizada del siguiente modo:

1. Si el argumento del trasfondo es correcto, entonces no podemos atribuir estados mentales irracionales a menos que el sujeto sea ampliamente racional (esto es, a menos que su patrón de estados mentales y acciones conserve un trasfondo de racionalidad).

2. Podemos atribuir estados mentales irracionales a sujetos que no son "ampliamente racionales" (que no conservan un trasfondo general de racionalidad). El caso de los delirios lo prueba.

3. Por lo tanto, el argumento davidsoniano es falso.

El desafio para Bortolotti es mostrar la verdad de la segunda premisa, esto es, que efectivamente podemos atribuir estados irracionales (por ejemplo: una creencia delirante) a sujetos que no son ampliamente racionales o que no conservarian un trasfondo general de racionalidad. Su estrategia se compone de dos pasos: (i) aceptar que los sujetos creen genuinamente los contenidos que afirman, y (ii) defender que no es legitimo sostener que estos sujetos son ampliamente racionales (o que preserven el trasfondo de racionalidad).

En cuanto al primer punto, Bortolotti suscribe explícitamente la concepción doxástica de los delirios, según la cual estos últimos deben ser vistos como creencias ${ }^{18}$. A su entender, debemos aceptar que algunos sujetos delirantes tienen creencias con determinados contenidos mentales, aunque estos últimos puedan ser bizarros, dado que los sujetos pueden ser vistos como auténticos creyentes. Por ejemplo: cuando actúan según su delirio, cuando ofrecen argumentos tentativos para justificarlos y cuando relacionan el contenido de su delirio con otras creencias que poseen. Un ejemplo de estas consideraciones es la paciente delirante "BC", quien cree que está siendo vigilada en su hogar a través de unos dispositivos de alta tecnología. BC actuó según su delirio cuando gastó £300 para que revisaran y sacaran de su hogar los dispositivos. Como argumento

\footnotetext{
${ }^{17}$ Ibid., p.1. La traducción es mía.

${ }^{18}$ Cf. Bortolotti, L., Delusions and Other Irrational Beliefs, Nueva York: Oxford University Press, 2009, p. 3 y también "In Defence of Modest Doxasticism About Delusions", en: Neuroethics, v. V (2011), pp. 39-53.
} 
tentativo para justificarlo, sostenía que escuchaba sus conversaciones repetidas en el trabajo y en el transporte público y esto la convencía de que estaba siendo vigilada. Asimismo, relacionaba su delirio con otras creencias, por ejemplo, con la creencia de que a la policía no le interesaba su caso. Como consecuencia, se inscribió en un curso de detective ${ }^{19}$. Acuerdo en este punto con Bortolotti, ya que como el mismo Davidson sostiene, "si sabemos que una afirmación es sincera, y que las palabras, que [el sujeto] dijo en esa ocasión, significan algo, casi siempre vamos a estar en lo cierto al suponer que el hablante cree lo que ha afirmado" 20 . De acuerdo a esto, resultaría adecuado considerar a los delirios de estos sujetos como creencias, dado que esto permitiría dar sentido a sus pensamientos y comportamientos. Como señala Bortolotti, cuando consideramos que estos sujetos creen aquello que afirman, podemos -en buena medida- explicar y predecir su comportamiento ${ }^{21}$.

A continuación, Bortolotti sostiene que el argumento del trasfondo falla ya que algunos casos de delirios pueden ser descritos como creencias (irracionales), a pesar de la ausencia de un trasfondo de racionalidad. Para apuntalar esta afirmación, Bortolotti presenta algunas consideraciones en contra de la idea de que los sujetos delirantes puedan ser considerados como ampliamente racionales, tal como exigiría el requisito davidsoniano para la atribución intencional. En particular, Bortolotti afirma que el davidsoniano interesado en defender que podemos considerar a los delirios como creencias irracionales y atribuírselas a los agentes en cuestión, debería mostrar que el patrón de pensamientos y comportamientos de estos sujetos es "en gran medida" racional, para poder así sostener que se halla presente el trasfondo de racionalidad que permite la atribución. Es decir, debería mostrar que estos fenómenos constituyen infracciones a la racionalidad de carácter temporal y local, tales como los errores de razonamiento ordinario. No obstante, Bortolotti considera que tal interpretación es insostenible en la medida en que hay evidencia en contra de la idea de que los sujetos delirantes puedan ser considerados como "ampliamente" racionales ${ }^{22}$. A la hora de mostrar que este es el caso, examina un delirio monotemático: el del síndrome de Capgras. Por ello, antes de avanzar con la reconstrucción y

${ }^{19}$ Cf. Fulford, B., Oxford Textbook of Philosophy and Psychiatry, Nueva York: Oxford University Press, 2006, p.163.

${ }^{20}$ Cf. Davidson, D., Mente, mundo y acción: claves para una interpretación, Barcelona: Paidós, p. 68.

${ }_{21}$ Cf. Bortolotti L., "In Defence of Modest Doxasticism About Delusions", en: Neuroethics, v. V (2011), p. 43.

${ }^{22}$ Ibid. 
evaluación de sus argumentos me detendré a describir brevemente el tipo de delirio que se manifiesta en este síndrome.

\section{El sindrome de Capgras}

El síndrome de Capgras fue descrito por primera vez en 1923 por Capgras y Reboul-Lachaux bajo la denominación de "l'illusion des sosies" "iilusión de dobles"), como un cuadro de falsa identificación delirante ${ }^{23}$ que consiste en la creencia de que una persona, generalmente cercana y afectivamente significativa, ha sido sustituida por un doble, quien es considerado un impostor. Implica la convicción de que el familiar, aunque idéntico al real, es una persona diferente ${ }^{24}$.

El delirio de Capgras es el más frecuente de los síndromes de falsa identificación delirante. Es un delirio restringido, generalmente, a una persona particular, aunque en algunos casos puede llegar a involucrar a varias. Presenta una duración variable y puede tratarse de un fenómeno transitorio o crónico ${ }^{25}$. Un fenómeno de naturaleza similar, que puede en algunos casos coexistir con delirio de Capgras, es la prosopagnosia, una alteración que incapacita al paciente a reconocer visualmente los rostros, tanto de sí mismo como de sus familiares ${ }^{26}$.

Se ha documentado que este delirio se presenta tanto en trastornos específicamente psiquiátricos (como la esquizofrenia) como en trastornos neurológicos (en demencias como el mal de Alzheimer y Parkinson, y otras lesiones cerebrales). Independientemente del diagnóstico, el cuadro clínico de la mayoría de los pacientes que desarrolla un delirio de Capgras está dominado por un componente paranoide ${ }^{27}$. A continuación presentaremos un caso, a modo de ejemplo, que representa las principales características de este sindrome.

23 Cf. Madoz Gúrpide, A. y R. Hillers-Rodriguez, "Delirio de Capgras: una revisión de las teorías etiológicas", en: Revista Neurología, v. L (2010), p. 420.

24 Ibid.

${ }^{25}$ Cf. Breen, N., Caine, D., Coltheart, M., Hendy, J., y C. Roberts, "Towards an Understanding of Delusions of Misidentification", en: Mind \& Language, v. XV (2002), pp. 74-110.

${ }^{26}$ Por ser fenomenológica y anatómicamente opuestos, se ha considerado que un cuadro es la imagen especular del otro: en el sindrome de Capgras el paciente preserva la capacidad para reconocer rostros, pero falla la sensación de familiaridad, al contrario que en la prosopagnosia. Se distinguen además por el carácter altamente selectivo del delirio de Capgras, ausente en la prosopagnosia (Cf. Madoz Gúrpide, A. y R. Hillers-Rodriguez, o.c., p. 420.)

${ }^{27}$ Ibid., p. 422. 


\section{1. El caso DS}

Hirstein y Ramachadrán ${ }^{28}$ han presentado el caso de "DS", un paciente con sindrome de Capgras quien afirma que sus padres son impostores (teniendo un delirio más marcado hacia su padre que a su madre). DS es un hombre brasilero que ha estado internado en un hospital debido a un accidente de tráfico que le causó una fractura parietal. Aproximadamente un año después de su accidente, ya recuperado, DS fue llevado por sus padres a consulta médica porque decía verlos a ambos como impostores. Se le realizaron exámenes diagnósticos y no se hallaron deficiencias en funciones complejas, ni había señales de demencia. Cuando el médico (M) le preguntaba a DS por qué decia que su padre era un impostor, DS respondia: "él se ve exactamente como mi padre, pero no es mi padre", "él es un buen hombre pero no es mi padre" 29 . En un diálogo entre M y DS, este último afirmaba ${ }^{30}$ :

- M: ¿Por qué ese hombre finge ser tu padre?

- DS: Esto es tan sorprendente doctor, ¿por qué alguien pretendería ser mi padre? Quizás mi padre empleó a alguien para que me cuide y le dio algún dinero para poder pagar mis cuentas.

Al tiempo, su padre probó un simple "truco" para intentar ayudar a DS. Se apareció de repente en su habitación y le dijo: "el hombre que te ha estado cuidando estos días es un impostor, él no es realmente tu padre. Lo he enviado de regreso a China. Yo soy tu verdadero padre. ¡Es tan bueno verte de nuevo, hijo!"31. Luego de este episodio, el delirio de DS pareció disminuir ligeramente como se evidencia en el siguiente diálogo ${ }^{32}$ :

- M: ¿Quién es el hombre que te trajo hoy?

- DS: Mi padre.

- M: Entonces ¿quién estuvo cuidando de ti antes?

- DS: Aquel hombre ha vuelto a China. Él lucía muy similar a mi padre, pero ya se ha ido.

Unas semanas después, DS volvió manifestar el delirio, afirmando que el impostor había regresado de su viaje a China. Un tiempo después reconoció

\footnotetext{
${ }^{28}$ Cf. Hirstein, W. y V. Ramachadrán, "Capgras Syndrome: a Novel Probe for Understanding the Neural Representation of the Identity and Familiarity of Persons”, en: Biological Sciences, v. CCLXIV (1997), pp. 437-444.

${ }^{29}$ Ibid., p. 438. La traducción es mía.

30 Ibid.

${ }^{31}$ Ibid., p. 439.

32 Ibid.
} 
que estaba comenzando a aceptar a este sujeto, aunque solo intelectualmente pero todavía no emocionalmente.

IV. Revisión de creencias: el síndrome de Capgras vs. la falacia de conjunción

Bortolotti invita al lector a comparar dos casos imaginarios: uno de un sujeto con sindrome de Capgras (Mike) con otro de una joven (Julia) que comete un error de razonamiento "ordinario", en particular una falacia de conjunción ${ }^{33}$, y se pregunta en qué sentido esta joven puede ser tan racional (o irracional) como el sujeto que padece de Capgras. Esta comparación pretende llevarnos a aceptar que en los casos de Capgras la falla de racionalidad es distinta -en un sentido específico- a la falla de racionalidad que tiene lugar en el caso de la falacia y que, por ello, debemos concluir que el trasfondo de racionalidad se halla ausente en Mike.

Con el objetivo de establecer por qué esta clase de delirios, a diferencia de lo que ocurre con el caso de la falacia de conjunción, no satisfacen los requisitos davidsonianos para la atribución intencional ${ }^{34}$, Bortolotti enfatiza un elemento particular constitutivo de la racionalidad: la capacidad de revisar las propias creencias ${ }^{35}$. Luego, afirma que una de las condiciones que de acuerdo a Davidson se impone a los agentes intencionales, es que cuenten con la capacidad para restaurar la racionalidad, es decir para revisar sus creencias una vez que una desviación respecto a las normas de racionalidad ha sido señalada o reconocida. En sus palabras: "dentro de las condiciones que Davidson impone a los sistemas de intencionales, la capacidad para restablecer la racionalidad desempeña un papel central. Para Davidson, es una condición de los sistemas intencionales que estén dispuestos a revisar sus creencias con el fin de ajustarse a las normas de la racionalidad, una vez que su desviación de la racionalidad se le ha señalado"36.

En el caso del delirio de Capgras, Mike cree que su esposa ha sido reemplazada por una impostora, y en el caso del error de razonamiento ordinario Julia comete una falacia lógica cuando estima, para la probabilidad de la conjunción

\footnotetext{
${ }^{33}$ Cabe señalar que Bortolotti se limita a presentar estos casos de carácter hipotético. No se detiene, en cambio, a evaluar casos clinicos reales ( $C f$. Bortolotti, L., "Intentionality Without Rationality", en: Proceedings of the Aristotelian Society, CV (2005), pp. 369-376).

${ }^{34} \mathrm{Sin}$ embargo, Bortolotti considera que estos casos de delirios podrian ser aun explicados con éxito bajo la "actitud intencional" (Cf. Ibid.).

35 Ibid.

${ }^{36}$ Ibid., p. 389. La traducción es mía.
} 
de dos sucesos, un mayor valor que el estimado para la probabilidad simple de uno de los sucesos. Bajo una lectura "superficial", sostiene Bortolotti, puede decirse que ambos casos se apartan de las normas de racionalidad solo en dos puntos: las creencias con respecto a la identidad del cónyuge en el primer caso y las creencias con respecto a la probabilidad de conjunción de ciertos acontecimientos en el segundo. Sin embargo, señala que Mike y Julia no son irracionales de la misma manera, ya que difieren en un punto nodal: mientras que seguramente Julia estaria dispuesta a rectificar su error y recobrar la racionalidad de sus creencias sobre la conjunción cuando un profesor le indique su error, dificilmente Mike abandonaria la creencia de que su esposa es una impostora si el terapeuta se lo señalara ${ }^{37}$. Al respecto, dice que "aunque a ellos pueden atribuirse creencias, los sujetos delirantes no pueden considerarse como racionales. Sus creencias son siempre enérgicamente resistentes al cambio"38. La autora agrega: "el comportamiento típico de los sujetos delirantes sugiere que no satisfacen los criterios de caracterización intencional, de acuerdo con la mejor lectura de lo que cuenta como trasfondo de racionalidad. A criterio de Bortolotti, el caso de los delirios proporciona una razón para cuestionar la idea aceptada de que no puede haber intencionalidad sin racionalidad"39.

En concreto, la autora argumenta que esta resistencia a la revisión de creencias por parte de los sujetos delirantes hace que deban ser vistos como carentes de un trasfondo general de racionalidad (algo que no ocurre, a su criterio, con quienes cometen una falacia de conjunción). En otras palabras, que la falla de racionalidad exhibida por estos sujetos delirantes es de un tipo específico, ya que no solo son incapaces de integrar sus creencias de modo coherente sino que, además, fallan al revisarlas cuando tienen evidencia en contra de las mismas.

Finalmente, Bortolotti señala que si, pese a todo, aún quisiéramos considerar que el sujeto delirante es "ampliamente racional" aduciendo que este todavia mantiene algunos vínculos racionales con otras creencias y acciones, no solo estariamos trivializando lo que consideramos "racional", sino que además "careceríamos de recursos para discriminar entre el comportamiento de los creyentes que pueden recuperarse de los fallos de racionalidad y los que no

\footnotetext{
37 Ibid., p. 390.

38 Ibid., p. 392.

39 Ibid.
} 
pueden" ${ }^{40}$. Esta distinción, a su criterio, juega un papel clave en la caracterización de Davidson del argumento del trasfondo. Así, luego de establecer estas consideraciones, mediante las cuales pretende haber mostrado que el sujeto que padece del delirio de Capgras tiene una creencia irracional ("creo que mi esposa es una impostora") y a la vez carece de un trasfondo de racionalidad en el patrón de sus estados mentales y acciones (en particular por fallar en revisar sus creencias), Bortolotti concluye que el argumento davidsoniano es falso.

\section{Una defensa al enfoque davidsoniano}

Las grandes desviaciones de las normas fundamentales de la racionalidad son más propensas a estar en el ojo del intérprete que en la mente del interpretado Donald Davidson ${ }^{41}$

Bortolotti asume que, para Davidson, tener un trasfondo general de racionalidad implica llevar a cabo procesos adecuados de revisión de creencias. Pero, según su razonamiento anterior, parece implícitamente suponer un requisito más fuerte que aquel que exige el propio Davidson. Para ver este punto con más detalle, reconstruiremos su argumentación previa:

1. Ser un sujeto "ampliamente racional", es decir, conservar un trasfondo de racionalidad en el patrón de estados mentales, implica ser capaz de revisar adecuadamente todas las creencias.

2. El sujeto que padece de síndrome de Capgras falla en la revisión de algunas de sus creencias: no corrige sus creencias delirantes.

3. Por lo tanto, el sujeto carece de un trasfondo general de racionalidad.

A continuación, cuestionaré el argumento esgrimido por Bortolotti. Para ello, en primer lugar, reconsideraré la segunda premisa y argumentaré que el sujeto que padece de Capgras no falla realmente en revisar sus creencias (V.1). Si esta estrategia no lograse todavía convencer al lector, presentaré otro argumento independiente para objetar la primera premisa de Bortolotti. En función de esto último, recurriré a la tesis de la compartimentación de lo mental de Davidson con el objetivo de mostrar que la posición de nuestro filósofo, adecuadamente interpretada, no demanda exactamente lo mismo que la primera premisa de

\footnotetext{
${ }^{40}$ Bortolotti, L., "Delusions and the Background of Rationality", en: Mind and Language, v. XX (2005), p. 204.

${ }^{41}$ Davidson, D., Problems of rationality, Nueva York: Clarendon Press, 2004, p. 204.
} 
la objeción de Bortolotti (V.2). A partir de estas consideraciones, concluiré que tenemos buenas razones para no aceptar, al menos para el caso del delirio de Capgras, la conclusión esgrimida por Bortolotti.

\section{V.1. Re-examinando el delirio de Capgras}

En lo que sigue pretendo responder la postura de Bortolotti sobre la incapacidad de los sujetos delirantes (como aquellos que padecen el síndrome de Capgras) para recuperarse de sus violaciones a las normas de racionalidad (núcleo de su argumento en contra de la persistencia de un trasfondo de racionalidad en sujetos que padecen de delirios). ¿Qué puede decir un davidsoniano sobre la resistencia del sujeto para modificar sus creencias delirantes cuando se le presenta evidencia que la desacredita? Considero que podrían brindarse al menos dos tipos de respuestas. Detengámonos ahora en el primero.

Un primer tipo de respuesta parte de poner en cuestión que, en el caso discutido por Bortolotti, la creencia del sujeto delirante y la supuesta evidencia disconfirmatoria representen efectivamente una violación explícita a las normas de racionalidad davidsonianas. A fin de desarrollar esta linea argumentativa, me valdré de la interpretación que Pacherie ${ }^{42}$ ha defendido sobre el delirio de Capgras. Partiendo de la pregunta sobre qué tipo de información contaría como evidencia legítima en contra de la creencia delirante del sujeto que padece delirio de Capgras, argumentaré que si consideramos el carácter peculiar de la experiencia del sujeto veremos que, aunque a simple vista parece irracional, de hecho no incurre en una violación explícita de racionalidad. Para ello, reconstruiré un modelo plausible que da cuenta de la formación del delirio de Capgras y posteriormente cuestionaré cuál es el peso que debiéramos dar a la presunta evidencia disconfirmatoria en estos casos.

\subsubsection{Sobre la formación de la creencia delirante}

A partir de una revisión de modelos recientes sobre el procesamiento visual de rostros, y la forma en que estos dan cuenta de la generación de respuestas afectivas ante rostros conocidos, Pacherie sostiene que el sistema subyacente a los sentimientos de familiaridad (y no-familiaridad) que experimentamos ante rostros bien conocidos es de carácter modular ${ }^{43}$. En función de esto, afirma que el delirio presente en el síndrome de Capgras (en particular

\footnotetext{
${ }^{42} C f$. Pacherie, E., "Perception, Emotions and Delusions: Revisiting the Capgras Delusion", en: Bayne, T. y J. Fernandez (eds.), Delusions and Self-Deception, Hove: Psychology Press, 2008, pp. 105-123.

${ }^{43}$ Para más detalles de esta propuesta modularista, cf. ibid., p. 106.
} 
su formación y persistencia) puede ser explicado por la presencia de fallos en este sistema modular emocional y que la experiencia de no-familiaridad que vive el paciente forma parte del contenido mismo del delirio.

Uno de estos modelos de procesamiento visual de rostros, prevaleciente en la literatura actual, es el desarrollado por Haxby y colegas ${ }^{44}$. A partir del estudio con imágenes cerebrales funcionales, los neurocientíficos concluyeron que el lóbulo temporal presentaría dos vías para el procesamiento de rostros: una vía temporal medial, que involucra el giro fusiforme, y una vía temporal lateral, que involucra el surco temporal superior. En función de esta evidencia, han conjeturado diferentes especializaciones funcionales para ambas vías: la vía medial se especializaría en la representación de los aspectos invariantes de los rostros que subyacen a la identidad personal, mientras que la vía lateral produciría representaciones de los aspectos cambiantes de los rostros. De este modo, el modelo distingue entre un sistema de procesamiento central y un sistema de procesamiento extendido 45 .

El modelo de Haxby y colegas podría proporcionar las bases para una teoria mindreading del delirio de Capgras (y de otros delirios de falsa identificación). La vía temporal medial produciría "representaciones externas", es decir, las representaciones de la apariencia fisica del rostro de una persona. Por el contrario, la vía temporal lateral produciría información pertinente a las "representaciones internas" de una persona, es decir, representaciones sobre cómo es su mente. En particular, la percepción de aspectos cambiantes de los rostros proveería información sobre el estado actual de la mente de la otra persona. La mirada, por ejemplo, podría informarnos sobre aquello a lo que la persona está atendiendo, y qué intereses actuales tiene, así como la expresión facial nos podría informar sobre el estado emocional de la persona. De acuerdo al modelo de Haxby y colegas, en el sindrome de Capgras, la vía temporal lateral no funcionaría correctamente y como consecuencia se producirian fallos en la producción de una "representación interna" o se produciría una representación del rostro diferente a la que el sujeto con Capgras utilizaba

\footnotetext{
44 Cf. Haxby, J., Hoffman, E. y I. Gobbini, "The Distributed Human Neural System for Face Perception”, en: Trends in Cognitive Science, v. IV (2000), pp. 223-233. En particular, me centraré aquí en la interpretación realizada por Hirstein de este modelo, a los fines de iluminar por qué podría producirse, desde la experiencia de no-familiaridad que vive el sujeto con Capgras, la creencia de que su familiar es un impostor. Cf. Hirstein, W., Brain Fiction, Cambridge: MIT Press, 2005, p. 133.

45 Ibid., p. 425.
} 
previamente ${ }^{46}$. Estas hipótesis resultan coherentes con la evidencia clínica que refiere que algunos pacientes con Capgras denuncian diferencias psicológicas entre el sujeto "original" y el impostor ${ }^{47}$.

Hirstein $^{48}$ afirma que el deterioro de la vía temporal lateral podría no solo interrumpir la lectura correcta de las expresiones de emociones sino también interrumpir la identificación de la "firma dinámica" del rostro de la persona ${ }^{49}$. Alguien con un impedimento tal no solo confundiria, por ejemplo, una expresión de preocupación con una expresión de enojo, sino que además vería a esta expresión de enojo como diferente en sus dinámicas (diferente a la manera en que usualmente esta persona expresa el enojo). Como Hirstein señala, de acuerdo con este punto de vista, el paciente que padece de Capgras "está mirando a alguien que visualmente se parece a su padre, pero que parece tener una mente diferente, una personalidad diferente, con diferentes disposiciones a hacer cosas diferentes. Esto es exactamente lo que un impostor es, y esta es exactamente la experiencia que uno tendría mientras mira al impostor"50.

La sensación de familiaridad vendría unida a un objeto, el rostro, el cual es visto como una ventana a la personalidad de alguien (una "representación interna") más que como una "representación externa". En otras palabras, la sensación de familiaridad vincula a un rostro, simplemente concebido como una configuración particular de características fisicas, con una personalidad. Entonces, si el contenido de la experiencia del paciente puede ser concebido, como Hirstein lo describe, como una experiencia de la persona visualmente presentada como siendo no-familiar, entonces la creencia del impostor, lejos de ser una explicación absurda y fantástica de la experiencia anormal, sería "una lectura directa de la misma" 1.

\footnotetext{
46 Ibid.

47 Cf. Young, A., Face and Mind, Oxford: Oxford University Press, 1998, p. 39.

48 Cf. Hirstein, W., o.c.

49 La firma dinámica estaría constituida por los movimientos característicos o idiosincrásicos que un rostro particular hace (tales como la sonrisa distintiva o la forma de expresar la sorpresa). O’Toole y colegas han señalado que la familiaridad con un rostro permite a uno extraer su firma dinámica y afirman además que esta información sería utilizada, junto con representaciones de características invariantes, para la identificación de rostros de familiares $(C f$. O’Toole, A., Roark, D. y H. Abdi, "Recognizing Moving Faces: a Psychological and Neural Synthesis", en: Trends in Cognitive Sciences, v. VI (2002), pp. 261-266).

${ }^{50}$ Cf. Hirstein, W., o.c., p. 133. La traducción y la cursiva son mías.

51 Cf. Pacherie, E., o.c., p. 114.
} 


\section{V.1.2. Sobre la revisión de la creencia delirante}

Pacherie señala, además, que la modularidad del sistema que produce la sensación de familiaridad (y no-familiaridad) podría contribuir a explicar por qué las creencias delirantes se mantienen firmemente. Sugiere que en los procesos de revisión de creencias un sujeto debería valerse de: (i) sus conocimientos previos: (a) su conocimiento biográfico, (b) su conocimiento general (o "enciclopédico") sobre el mundo, (ii) el testimonio de otros agentes, (iii) la búsqueda de nueva evidencia ${ }^{52}$. Si aplicamos esto a los delirios de Capgras, podremos reconsiderar el peso de los distintos tipos de evidencia con los que cuentan quienes sufren de esta patologia:

(i) Uso de conocimientos previos: (a) conocimiento biográfico: existe un conocimiento biográfico relativo a la relación entre el paciente y el familiar/ impostor, que refiere a episodios comunes compartidos. Ahora bien, una confrontación del paciente con el supuesto impostor, en donde este último relate hechos sobre experiencias compartidas para mostrar que él o ella es quien dice ser y no un impostor, posiblemente no será tomada como evidencia determinante de que tal sujeto no es un impostor. Más bien, esta evidencia suele ser considerada como evidencia de que el sujeto es un "buen impostor", ya que un impostor no solo es alguien que es similar visualmente al otro sujeto (un "sosie"), sino más bien alguien que se hace pasar por otra persona y que pretende que otro crea esto. Además, como Pacherie destaca, es posible que al hablar con su familiar, el paciente con Capgras experimente una inquietante sensación de no-familiaridad, junto quizás con la impresión de que esta otra persona es mal intencionada, lo cual reforzará su creencia ${ }^{53}$. Por lo tanto, el uso del conocimiento biográfico podría ser tomado, razonablemente, no como evidencia disconfirmatoria sino, por el contrario, como una confirmación de que la persona que se parece a la esposa de uno está tratando de hacerse pasar por ella y por lo tanto, es un impostor. (B) conocimiento del mundo: los pacientes con Capgras pueden ser capaces de apreciar la inverosimilitud de las creencias sobre impostores ${ }^{54}$. El paciente "S" por ejemplo, cuando se le pregunta

52 Ibid., p. 117.

53 Ibid., p. 118.

54 Reimer ha puesto especial énfasis en este punto, destacando que muchos pacientes psiquiátricos delirantes se consideran a ellos mismos como una excepción a ciertas generalizaciones pertinentes. Por ejemplo, suelen afirmar expresiones tales como "generalmente las historias de impositores son inverosímiles, pero mi caso constituye una excepción” ( $C f$. Reimer, M., "A Davidsonian Perspective on Psychiatric Delusions", en: Philosophical Psychology, v. XXIV (2011), pp. 659-677 y Reimer, M., "Davidsonian Holism in Recent Philosophy of Psychiatry”, en: Preyer, G. 
cómo puede explicar lo que le está sucediendo relata: "No lo sé. He tratado de entender esto yo mismo y es prácticamente imposible. Si Ud. me dijera que no me cree sería perfectamente comprensible. De hecho, cuando cuento esta historia, siento que estoy inventando una historia"55. Pero esta consideración por sí sola puede que no tenga el peso suficiente para llevarlo a abandonar su creencia delirante, ya que inverosímil no es sinónimo de imposible. Por lo tanto, el uso de conocimiento general acerca del mundo podria ser tomado, también razonablemente, como una confirmación de que la situación a la que el sujeto se enfrenta es de hecho rara y que requiere una explicación, más que como una indicación de que la situación no es lo que el sujeto piensa que es.

(ii) Testimonio de otros agentes: Pacherie afirma que el testimonio de los demás funciona como parte de la división social del "trabajo epistémico"56. En la misma forma que para el lenguaje nos basamos en los hablantes "expertos" para conocer el significado exacto de ciertas palabras, para las creencias podemos basarnos también en expertos que nos dicen si debemos aceptar una creencia particular o rechazarla. Pero, por supuesto, quién cuenta como un experto depende de aquello sobre lo cual la creencia versa. Los expertos en los cuales se podría confiar para comprobar las creencias sobre las matemáticas no serian los mismos que se podrian consultar para creencias sobre gastronomía. Si lo vemos desde esta perspectiva, parece obvio que los extraños no contarán como expertos calificados para decirle a un sujeto quién es su conyugue o su padre. Sin embargo, otros familiares y amigos podrian calificar como expertos. Luego, ¿por qué los pacientes no atienden al testimonio provisto por ellos? Una primera respuesta posible a esta interrogante es que, para el sujeto, nadie es más idóneo que él mismo para saber quién es su cónyuge/su padre. En segundo lugar, si bien otros familiares y amigos podrían calificar como expertos, el paciente suele desestimar la opinión de estas personas porque en muchos casos termina sospechando que todos son impostores (y/o cómplices), ya que el delirio tiende a extenderse. Cualquiera sea el caso, parece que el sujeto tiene una justificación para tratar el testimonio de los otros agentes del modo en que lo hace, para terminar confiando únicamente en su experiencia, que le dice que la persona que se encuentra delante de él no es su familiar.

(ed.), Donald Davidson on Truth, Meaning, and the Mental, Oxford: Oxford, University Press, 2012, pp. 1-21).

${ }_{55}$ Alexander, M.; Stuss, D. y D. Benson, “Capgras Syndrome: A Reduplicative Phenomenon”, en: Neurology, XXIX (1979), p. 335.

${ }^{56}$ Cf. Pacherie, E., o.c., p. 119. 
(iii) Búsqueda de nueva evidencia: en el delirio de Capgras, la creencia delirante se basa en la experiencia inusual que tiene el sujeto cuando mira a sus familiares. Al respecto, realizar una búsqueda de evidencia adicional podría significar que el paciente se mantenga observando mirando repetidas veces el rostro de su familiar, para ver si se restaura la sensación de familiaridad. El problema, sin embargo, es que si la ruta afectiva de reconocimiento de rostros está dañada (como se hipotetiza), el procedimiento de comprobación seguiría dando el mismo resultado negativo. Es decir que esta evidencia adicional, en vez de ayudar a falsar la creencia, solo traería una nueva confirmación a favor de esta. En este sentido parece estar funcionando en estos sujetos una premisa implícita, como la siguiente: "si esta persona fuera mi familiar (mi padre o cónyuge en nuestros ejemplos) debería sentir aquel sentimiento de familiaridad que se me presenta cuando veo los rostros de mis familiares". Ante esta situación, el sujeto podría seguir buscando evidencia para ver si se restaura la sensación de familiaridad, pero el problema es que, como el canal de información (la ruta afectiva de reconocimiento facial) está dañado, el procedimiento de control sigue dando el mismo resultado negativo. Como Bermúdez ha señalado, en estos casos la repetición de la experiencia se traduce en un refuerzo de la creencia y no en su rechazo ${ }^{57}$.

En sintesis, la falla de un proceso afectivo (presumiblemente modular) involucrado en el reconocimiento de los rostros (en las expresiones emocionales, la identificación de firmas dinámicas, y la generación de respuestas autonómicas como los sentimientos de familiaridad) pueden permitir comprender las experiencias y creencias delirantes de los pacientes con Capgras. Como Pacherie sugiere, las creencias delirantes heredarian su contenido de la experiencia delirante de falta de familiaridad ${ }^{58}$.

$\mathrm{Si}$ este enfoque es adecuado, y es plausible asumir que la naturaleza particular de las creencias determina qué procedimientos de control son adecuados, puede verse que la razón por la cual los pacientes con Capgras fallan para desestimar sus creencias delirantes no es que ellos no puedan utilizar estos

${ }^{57}$ Cf. Bermudez, J-L., "Normativity and Rationality in Delusional Psychiatric Disorders", en: Mind \& Language, v. XVI (2001), pp. 457-493. Respecto a este punto, soy consciente de que alguien podría objetar que si el daño es en una vía visual, utilizando otra modalidad (por ejemplo: auditiva), se podría restaurar el sentimiento de familiaridad. Sin embargo, puede argüirse que en los seres humanos la modalidad visual tiende a dominar sobre otras modalidades sensoriales. Como Pacherie ha señalado, si un paciente cuando habla cara a cara con su padre no tiene el sentimiento de familiaridad, pero cuando lo escucha sí, el conflicto entre la modalidad visual y la modalidad auditiva probablemente se resolvería a favor de la modalidad visual ( $C f$. Pacherie, E., ibid., p. 119). 58 Ibid., p. 120. 
procedimientos de comprobación, más bien sucede que estos procedimientos no logran producir evidencia disconfirmatoria.

En sintesis, el análisis anterior nos permite argüir que el paciente de Capgras no es epistémicamente incompetente, sino que sería víctima de un círculo vicioso epistémico. Sin embargo, y afortunadamente, este círculo vicioso se limita a las creencias con un tipo específico de contenido y etiología (la creencia delirante), de ahí el carácter circunscrito del delirio. Entonces, si este análisis es plausible, podemos considerar que en los casos de delirio de Capgras, como el de Mike y DS, no se trata de que los sujetos fallen en restaurar la racionalidad, sino que no encuentran evidencia disconfirmatoria de peso como para verse obligados a revisar su creencia. Esto se debe al carácter peculiar de la vivencia en primera persona que los sujetos experimentan ${ }^{59}$. Con lo cual tendriamos buenas razones para desestimar la segunda premisa del argumento de Bortolotti.

Aun así, si esto no resultara convincente a un lector escéptico y se insistiera en que efectivamente estos sujetos fallan al revisar sus creencias (en un sentido relevante), considero que hay un segundo tipo de respuesta que puede brindarse en contra del argumento de Bortolotti. Esta consiste en aceptar que la imposibilidad del sujeto para restaurar la racionalidad involucra una desviación respecto a las normas de racionalidad, sin embargo, si el delirio es circunscripto (como ocurre en el caso del Capgras), esto no significaria un problema para el enfoque davidsoniano, ya que este acepta fallos de carácter local. Podemos acudir así, a los fines de criticar la primera premisa del argumento de Bortolotti, a la tesis de la compartimentación de lo mental que esgrime Davidson para lidiar con el problema de la irracionalidad.

\section{V.2. La tesis de la compartimentación de lo mental}

Cuando se ocupa de los problemas conceptuales que los casos de irracionalidad plantean para su enfoque de la interpretación intencional, Davidson se focaliza en un tipo particular de irracionalidad: aquella que tiene lugar cuando se presenta algún tipo de inconsistencia interna. En este contexto, en

\footnotetext{
59 Por otra parte, me gustaría señalar que los casos analizados por Bortolotti podrían no ser considerados como análogos, dado que presentan marcadas características que los diferencian. Me refiero en particular al compromiso claramente emocional involucrado en la creencia de Mike, que refiere a su familiar cercano, vs. la creencia puramente "intelectual" (o desapegada afectivamente) de Julia. Podría también haber un compromiso emocional vinculado a estas creencias que juegue un rol específico en la modificación de las mismas. Sin embargo, dejaremos en suspenso este asunto aquí ya que merecía una investigación adicional sobre el vínculo entre emociones, creencias y procesos de revisión de las mismas.
} 
el cual nuestro filósofo intenta buscar una explicación psicológica de cómo los creyentes pueden ser inconsistentes, introduce la idea de que la mente puede estar particionada. Al respecto, sostiene que la mente puede estar dividida en dos o más estructuras cuasi-independientes. Al interior de cada una de estas partes, los eventos mentales se relacionarian de modos que respeten los principios de la racionalidad. Sin embargo, un estado mental perteneciente a una parte podría vincularse causalmente con un estado mental de otra parte sin mantener vínculos racionales con él. La característica necesaria para que haya una estructura de este tipo es que una parte de la mente exhiba un mayor grado de racionalidad o coherencia que la que se atribuye al todo ${ }^{60}$. Es decir que, asumiendo que las parte tienen algún grado de independencia entre sí, es posible comprender cómo distintas partes son capaces de albergar inconsistencias entre sí y a la vez interactuar causalmente unas con otras. Lo único que se requiere, son elementos organizados, en cuyo interior haya un aceptable grado de coherencia y donde un elemento pueda operar sobre otro según la modalidad de la causalidad no racional. De este modo, Davidson logra resguardar la coherencia como requisito del pensamiento, y a la vez dar cuenta de cómo el fenómeno de la irracionalidad es posible.

Recordemos, además, que en este contexto Davidson sostuvo que no tenemos problemas en comprender perturbaciones menores cuando hay un trasfondo de racionalidad que en gran medida compartimos, y que el hecho de que alguien actúe en algunas ocasiones de manera irracional no pone en peligro la capacidad para considerarle un sujeto intencional ni para atribuirle estados mentales. Aunque un pensamiento o acción no siga los principios de racionalidad (como podría ocurrir en el caso de la creencia del sujeto delirante), en tanto la mayoría de sus otros pensamientos/acciones sí lo hagan, la inteligibilidad estará garantizada. Así, resulta plausible en este marco pensar que la creencia delirante se encuentra "compartimentalizada" en alguna de las partes de la mente, y que en un proceso global de revisión de creencias, esta parte resulta inmune a la evidencia proveniente de otras. Lo único que necesitamos, para seguir considerando al agente como ampliamente racional

\footnotetext{
${ }^{60}$ En este punto Davidson recoge la tesis freudiana de la división de lo mental pero, por su grado de abstracción respecto al funcionamiento de la mente, se distancia de Freud, ya que no hace ninguna afirmación sustancial respecto a la naturaleza de tales divisiones. Simplemente se limita a defender la tesis de la compartimentación de lo mental, argumentando que es una tesis necesaria para poder explicar las formas típicas de irracionalidad (cf., ibid).
} 
(es decir, conservando un trasfondo de racionalidad), es que la mayoria de sus creencias, intenciones y acciones manifiesten los principios de racionalidad.

\section{V.3. ¿Trasfondo de racionalidad conservado?}

En lo que sigue, intentaré mostrar que en los casos de delirios de Capgras, el trasfondo de racionalidad en el patrón de estados mentales y comportamientos de estos sujetos puede considerarse conservado. Para ello, analizando el caso de Mike y DS, me concentraré en los requisitos de coherencia y correspondencia que demanda el enfoque davidsoniano.

Respecto a la coherencia podemos decir que existe coherencia entre la creencia de Mike "mi esposa ha sido reemplazada por un impostor" y otras creencias suyas, como podrian ser las siguientes: "quien dice ser mi esposa es una persona peligrosa", "la impostora sabe imitar muy bien a mi esposa", "la impostora le ha robado la ropa a mi esposa", etcétera. Asimismo, en el caso de $\mathrm{DS}$, hay coherencia entre su creencia "el hombre que me ha estado cuidado estos dias es un impostor" y su otra creencia "aquel hombre ha vuelto a China, lucía similar a mi padre pero no lo era"61.

Tampoco parecer haber en el delirio de Capgras ninguna inconsistencia explícita (es decir, una situación en la cual el sujeto acepte A y $\neg \mathrm{A}$ al mismo tiempo). La creencia de Mike posee el siguiente contenido: " mi esposa ha sido reemplazada por un impostor". Para que estemos en presencia de una inconsistencia explicita Mike debería aceptar también, y al mismo tiempo la siguiente creencia: "mi esposa (no) ha sido reemplazada por un impostor", y este no parece ser el caso. En ningún momento Mike acepta esta segunda creencia. De hecho, si nos focalizamos en el caso real que presentamos anteriormente, donde DS cree que el sujeto que luce como su padre es un impostor, vemos que DS solo sostiene en distintos momentos y frente a distinta evidencia que la persona que lo cuida es y no es, respectivamente, su padre. Tampoco aquí parece haber ninguna inconsistencia explícita. Entonces, es plausible pensar que, al menos en principio, en estos casos no parece haber inconsistencias internas del tipo especificado por Davidson.

En cuanto a la correspondencia, si bien las creencias delirantes de Mike y DS son -por hipótesis- falsas, probablemente la gran mayoría de sus otras

\footnotetext{
${ }^{61}$ Alguien podría objetar que las creencias " mi esposa ha sido reemplazada por un impostor" $\mathrm{y}$ " $m i$ padre ha sido reemplazado por un impostor" pueden no resultar coherentes con otras creencias generales tales como "las historias de impostores son inverosimiles". Sin embargo, no es obvio que estos sujetos acepten una creencia tal, e incluso si la aceptasen (como sucede en algunos casos), pueden escapar a la inconsistencia explícita afirmando que su caso constituye una excepción.
} 
creencias sean verdaderas. En el caso de Mike, podría tener creencias verdaderas tales como las siguientes: "tengo una esposa", "mi esposa luce de un modo particular X", "existe el casamiento", etcétera. En el caso de DS: "existe un país llamado China", "estuve internado en el hospital", "tengo un padre", etcétera. Es decir, compartirian con nosotros un número suficiente de creencias básicas que sustentarian la atribución de sus creencias delirantes.

Si estas consideraciones son plausibles, el trasfondo de racionalidad, en el sentido davidsoniano, puede considerarse conservado (al menos en este tipo de delirios). Recordemos además que, como bien señaló Davidson, ser racional es simplemente tener actitudes proposicionales "sin importar cuán confusas, contradictorias, absurdas, injustificadas o erróneas puedan ser estas" 62 , y el punto importante es que "la excepción (como desviación de las normas de racionalidad) no puede ser la regla"b3. Así, mientras el resto de creencias, actitudes y acciones del sujeto se atengan a los principios de racionalidad, tenemos buenas razones para considerar que ese sujeto posee el trasfondo de racionalidad necesario para que tenga lugar la atribución intencional. De este modo, sigue siendo cierta la afirmación davidsoniana de que las atribuciones de actitudes proposicionales implican atribuciones de racionalidad, garantizándose así la inteligibilidad.

Por otra parte, pienso que este modo de interpretar la tesis del trasfondo no resulta trivial por no permitirnos distinguir-como esgrime Bortolotti-casos de irracionalidad transitoria/ordinaria y crónica/patológica. Después de todo, no parece haber buenas razones para pedirle al davidsoniano que su teoría tenga la carga de proporcionar criterios que permitan distinguir entre casos normales y patológicos ni tampoco pienso que el argumento del trasfondo deba interpretarse de tal manera que ilumine la distinción entre casos crónicos (patológicos) o transitorios (comunes) de irracionalidad. Por supuesto, esto no significa negar la necesidad de dar cuenta de la distinción que señala Bortolotti entre casos normales y patológicos. Sin embargo, considero que los criterios para tal distinción posiblemente se encuentren relacionados con otros factores adicionales y no considerados en la obra davidsoniana, tales como el rol de las emociones y la importancia de determinadas competencias cognitivas necesarias para la interacción social. Por otra parte, esta lectura de la idea de trasfondo tampoco anula la posibilidad de distinguir los casos de sujetos racionales -con

${ }^{62}$ Davidson, D., Subjective, Intersubjective Objective, Oxford: Oxford Claredon Press, 2001, p. 142. La traducción es mía.

${ }^{63}$ Ibid., p.70. La traducción y la cursiva son mías. 
algunos fallos de carácter local- de aquellos casos graves de irracionalidad. Estos últimos casos son reconocidos -precisamente como señala el mismo Davidson- porque frente a ellos la interpretación se ve socavada.

Respecto a este último punto, es preciso señalar la siguiente tensión que subyace a la estrategia de Bortolotti. Por un lado, asume la tesis de la continuidad, según la cual las fallas de racionalidad (o la irracionalidad) de las creencias delirantes solo presentan diferencias de grado, y no de tipo, con las fallas de racionalidad de las creencias ordinarias. Por otro lado, para atacar las visiones racionalistas, como la de Davidson, Bortolotti destaca que el tipo de falla de racionalidad de los casos de delirios (como el de Capgras) es distinto en un sentido específico que nos previene de caracterizar al sujeto como ampliamente racional. Con lo cual parece sugerir, que la diferencia es cualitativamente distinta y que por, esto mismo, no es legítimo considerar a los sujetos que sufren de Capgras como conservando un trasfondo de racionalidad. Incluso, aun si quisiera aceptar que la diferencia entre creencias delirantes y creencias ordinarias es solo cuantitativa (solo que en un grado significativo) Bortolotti seguiria careciendo del criterio que reclama para distinguir entre casos de irracionalidad ordinaria de aquellos de irracionalidad patológica, ya que no resulta claro cuál sería el límite que trazaría tal distinción.

Ante esta encrucijada caben dos alternativas. Una es asumir una estrategia como la que he estado sugiriendo, según la cual una visión detenida de los delirios nos muestra que no son tan irracionales y que los sujetos que los padecen aún conservan el trasfondo de racionalidad, precisamente porque la diferencia con otras creencias en este aspecto no es categorial. Otra opción es abandonar su tesis de la continuidad, pero esto podría tener consecuencias indeseables para Bortolotti, dado que su proyecto teórico respecto a la irracionalidad, consiste fundamentalmente en defender la continuidad entre creencias ordinarias y creencias delirantes.

\section{Conclusiones}

En el presente artículo he reconstruido y objetado la crítica de Bortolotti al argumento del trasfondo davidsoniano, según el cual solo podemos atribuir contenidos mentales irracionales cuando estamos en presencia de un trasfondo general de racionalidad. Según vimos, Bortolotti ataca este argumento defendiendo la idea de que las creencias delirantes presentan una irracionalidad tal que no es posible considerar a los sujetos delirantes (en particular, a 
aquellos que padecen de síndrome de Capgras) como ampliamente racionales. No obstante, argumenta que es posible la atribución de dichas creencias en ausencia de un trasfondo de creencias, deseos e intenciones que se adecuen a los parámetros de racionalidad.

Al respecto, he criticado la segunda premisa del argumento de Bortolotti, señalando que el sujeto con síndrome de Capgras no falla -como a simple vista parece- en llevar a cabo un proceso racionalmente necesario de revisión de sus creencias. Para defender esta idea evalué el peso de la supuesta evidencia disconfirmatoria para el caso del delirio de Capgras. De acuerdo con la misma, un examen cuidadoso de algunos delirios monotemáticos, tales como el Capgras, nos brindan razones para pensar que no se trata de que el sujeto falle en restaurar la racionalidad sino que, debido a la misma enfermedad, no encuentra evidencia disconfirmatoria de peso como para verse obligado a revisar su creencia.

De modo independiente, la segunda estrategia consistió en objetar la primera premisa, recurriendo a la tesis de la compartimentación de lo mental de Davidson. Señalé que, mientras el delirio se encuentre circunscripto, el resto de pensamientos y acciones del sujeto conserve el trasfondo de racionalidad y el sujeto solo falle en revisar la creencia delirante, el enfoque davidsoniano aún no se encuentra en problemas ya que puede dar cuenta de fallos de este tipo mientras los mismos conserven un carácter local. A continuación, brindé razones por las cuales estos sujetos delirantes puede ser vistos (en un sentido no trivial) como conservando tal trasfondo de racionalidad. Nótese, entonces, que esta segunda estrategia, de modo independiente a la primera, consistió en objetar la primera premisa del argumento de Bortolotti, señalando que ésta demanda incluso más que el mismo requisito davidsoniano de racionalidad. Luego, procedí a argumentar que los delirios monotemáticos, tales como el Capgras, no representan un contraejemplo a la tesis davidsoniana sobre la atribución de estados intencionales.

Aunque coincido con el espíritu del trabajo de Bortolotti, al menos en la medida en que pretende mostrarnos las consecuencias negativas de idealizar nuestro nivel de racionalidad (y en particular el de nuestras creencias), difiero en que debamos negar el vínculo entre intencionalidad y racionalidad. Al respecto, considero que Bortolotti no ha presentado argumentos convincentes para que aceptemos dicha negación. Antes bien, se desprende del análisis realizado que aún tenemos buenas razones para pensar, junto a Davidson, que la racionalidad es una precondición para la atribución de estados mentales intencionales, 
y que esta constricción no implica -a diferencia de lo que Bortolotti parece sugerir-que no se puedan atribuir creencias irracionales. Antes bien, intenté mostrar, en defensa de Davidson, que esto es posible en tanto haya aún un trasfondo general de racionalidad compartido entre el sujeto y nosotros como intérpretes. Ahora bien, cabe señalar que he argumentado que para los casos de los delirios analizados, el trasfondo de racionalidad que exhiben los sujetos es suficiente, no obstante, restará realizar la tarea de determinar cuáles son los requisitos tanto suficientes como necesarios para decir que estamos en la presencia de racionalidad y si, en este sentido, la misma puede ser en algunos casos desligada de las atribuciones de intencionalidad. Como el mismo Davidson ha reconocido, este es un camino dificil en tanto exige no solo investigación empírica sino principalmente conceptual en tanto debemos decidir qué evidencias son relevantes para decidir si una criatura tiene una rica pauta de actitudes proposicionales (criterio considerado como suficiente para Davidson para detectar racionalidad).

En este sentido, aquí he defendido que para que el holismo de lo mental y el principio de racionalidad sigan siendo válidos, no se precisa que sean plenos, ya que el davidsoniano no tiene problemas para lidiar con los fallos de racionalidad que pueden presentar los sujetos delirantes, en la medida en que estos mantengan, como parece ocurrir en el caso del delirio de Capgras, un trasfondo de racionalidad compartido y sus perturbaciones no sean excesivas. Así, aun cuando algunos de sus pensamientos y/o acciones no se ajusten adecuadamente a las normas de racionalidad, mientras el resto sí lo haga, la interpretación se mantiene garantizada.

Un enfoque como el defendido nos permitirá además dar cuenta de nuestra capacidad para seguir considerando a determinados sujetos como agentes intencionales, atribuyéndoles estados mentales para dar cuenta de sus pensamientos y acciones, a pesar de que a simple vista, o bajo criterios de otra índole, parezcan irracionales. No obstante, es preciso reconocer que esto no significa que ningún delirio desafie el enfoque davidsoniano. Resta seguir investigando si esta defensa a Davidson que he delineado puede aplicarse a otro tipo de delirios, tales como los delirios politemáticos presentes en distintos tipos de esquizofrenia. 


\section{Bibliografia}

Alexander, M., Stuss, D. y D. Benson, "Capgras Syndrome: A Reduplicative Phenomenon", en: Neurology, v. XXIX (1979). https://doi.org/10.1212/wnl.29.3.334

Bermudez, J. L., "Normativity and Rationality in Delusional Psychiatric Disorders", en: Mind \& Language, v. XVI (2001), pp. 457-493. https://doi.org/ 10.1111/1468-0017.00179

Bortolotti, L., "Inconsistency and Interpretation", en: Philosophical Explorations, VI (2003), pp. 109-123. https://doi.org/10.1080/10002003058538743

Bortolotti, L., "Can We Interpret Irrational Behavior?", en: Behavior and Philosophy, v. XXXII (2004), pp. 359-375.

Bortolotti, L., "Delusions and the Background of Rationality", en: Mind and Language, v. XX (2005), pp. 189-208. https://doi.org/10.1111/j.0268-1064.2005.00282.x

Bortolotti, L., "Intentionality without Rationality", en: Proceedings of the Aristotelian Society, CV (2005), pp. 369-376. https://doi.org/10.1111/j.0066-7373.2004.00119.x

Bortolotti, L., Delusions and Other Irrational Beliefs, Nueva York: Oxford University Press, 2009. https://doi.org/10.1093/med/9780199206162.001.1

Bortolotti, L., "In Defence of Modest Doxasticism About Delusions", en: Neuroethics, v. V (2011), pp. 39-53. https://doi.org/10.1007/s12152-011-9122-8

Bortolotti, L., Delusions and Other Irrational Beliefs, Nueva York: Oxford University Press, 2009. https://doi.org/10.1093/med/9780199206162.001.1

Breen, N., D. Caine, M. Coltheart, J. Hendy y C. Roberts, “Towards an Understanding of Delusions of Misidentification", en: Mind \& Language, v. XV, (2002), pp. 74-110. https://doi.org/10.1111/1468-0017.00124

Campbell, J., "What Does Rationality have to Do with Psychological Causation? Propositional Attitudes as Mechanisms and as Control Variables “, en: Broome, M. y L. Bortolotti (eds.), Psychiatry as Cognitive Neuroscience: Philosophical perspectives, Oxford: Oxford University Press, 2009, pp. 137-149. https://doi. org/10.1093/med/9780199238033.003.0008

Davidson, D., “Truth and Meaning”, en: Synthese, v. XVII (1967), pp. 304-323. https:// doi.org/10.1007/BF00485035

Davidson, D., "How is Weakness of the Will Possible?", en: Feinberg, J. (ed.), Moral Concepts, Oxford: Oxford University Press, 1970, pp. 93-113.

Davidson, D., “Thought and Talk", en: Guttenplan, S. (ed.), Mind and Language, Oxford: Oxford University Press, 1975.

210 Davidson, D., "Two Paradoxes of Irrationality", en: Wollheim, R. y J. Hopkins (eds.), Philosophical Essays on Freud, Cambridge: Cambridge University Press, 1982, pp. 289-305. https://doi.org/10.1017/CBO9780511554636.018

Davidson, D., "A Coherence Theory of Truth and Knowledge", en: Henrich, D. (ed.), Kant oder Hegel?, Stuttgart: Klett-Cotta, 1983, pp. 154-173.

Davidson, D., "Incoherence and irrationality", en: Dialectica, v. XXXIX (1985), pp. 345354. https://doi.org/10.1111/j.1746-8361.1985.tb01603.x 
Davidson, D., “A Coherence Theory of Truth and Knowledge", en: Lepore, E. (ed.), Perspectives in the Philosophy of Donald Davidson, Oxford: Basil Blackwell, 1986, pp. 307-319.

Davidson, D., Inquiries into Truth and Interpretation, Oxford: Clarendon Press, 2001. https://doi.org/10.1093/0199246297.001.0001

Davidson, D., Subjective, Intersubjective Objective, Oxford: Oxford Claredon Press, 2001.

Davidson, D., Problems of Rationality, Nueva York: Clarendon Press, 2004. https://doi. org/10.1093/0198237545.001.0001

Dennett, D., The Intentional Stance, Cambridge: MIT Press, 1987.

Fulford, B., Oxford Textbook of Philosophy and Psychiatry, Nueva York: Oxford University Press, 2006.

Gerrans, P., "Cognitive Architecture and the Limits of Interpretationism", en: Philosophy, Psychiatry, \& Psychology, v. XI (2009), pp. 43-48. https://doi.org/10.1353/ ppp.2004.0039

Haxby, J., E. Hoffman y I. Gobbini. "The Distributed Human Neural System for Face Perception”, en: Trends in Cognitive Science, v. IV (2000), pp. 223-233. https:// doi.org/10.1016/S1364-6613(00)01482-0

Hirstein, W., Brain Fiction, Cambridge: MIT Press, 2005, p. 133.

Hirstein, W. y V. Ramachadrán, "Capgras Syndrome: a Novel Probe for Understanding the Neural Representation of the Identity and Familiarity of Persons", en: Biological Sciences, v. CCLXIV (1997), pp. 437-444. https://doi.org/10.1098/ rspb.1997.0062

Klee, R., "Why Some Delusions Are Necessarily Inexplicable Beliefs", en: Philosophy, Psychiatry \& Psychology, XI (2004), pp. 25-34. https://doi.org/10.1353/ ppp.2004.0044

Madoz Gúrpide, A. y R. Hillers-Rodriguez, "Delirio de Capgras: una revisión de las teorias etiológicas”, en: Revista Neurología, v. L (2010), pp. 420.

O’Toole, A., D. Roark y H. Abdi, "Recognizing Moving Faces: A Psychological and Neural Synthesis", en: Trends in Cognitive Sciences, v. VI (2002), pp. 261-266. https:// doi.org/10.1016/S1364-6613(02)01908-3

Pacherie, E., "Perception, Emotions and Delusions: Revisiting the Capgras Delusion", en: Bayne, T. y J. Fernandez (eds.), Delusions and Self-Deception, Hove: Psychology Press, 2008, pp. 105-123.

Reimer, M., "A Davidsonian Perspective on Psychiatric Delusions", en: Philosophical Psychology, v. XXIV (2011), pp. 659-677. https://doi.org/10.1080/09515089. 2011.562642

Reimer, M., "Davidsonian Holism in Recent Philosophy of Psychiatry", en: Preyer, G. (ed.), Donald Davidson on Truth, Meaning, and the Mental, Oxford: Oxford, University Press, 2012, pp. 1-21. https://doi.org/10.1093/acprof: oso/9780199697519.003.0012

Scarone, S., M. Manzone, O. Gambini, I. Kantzas, I. Limosani, A. D’Agostino y A. Hobson, "The dream as a model for psychosis: an experimental approach using bizarreness 
as a cognitive marker”, en: Schizophrenia Bulletin, v. XXXIV (2008), pp. 515-522. https://doi.org/10.1093/schbul/sbm116

Young, A., Face and mind, Oxford: Oxford University Press, 1998, p. 39. https://doi. org/10.1093/acprof:oso/9780198524205.001.0001

Recibido: 09/08/2015

Aceptado: 16/02/2016 